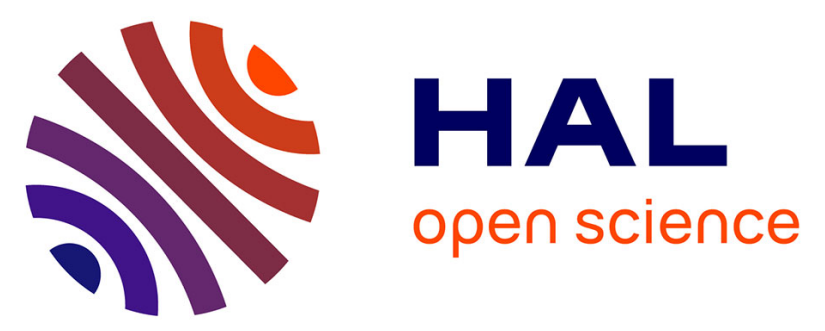

\title{
Analysis of Charge Transport and Ionization Effect in Space-Used Polymers Under High-Energy Electron Irradiation
}

\author{
T. Paulmier, B. Dirassen, D. Payan, M. Arnaout
}

\section{To cite this version:}

T. Paulmier, B. Dirassen, D. Payan, M. Arnaout. Analysis of Charge Transport and Ionization Effect in Space-Used Polymers Under High-Energy Electron Irradiation. IEEE Transactions on Plasma Science, 2017, 45 (8), p. 1933 - 1937. 10.1109/TPS.2017.2681180 . hal-01629220

\author{
HAL Id: hal-01629220 \\ https://hal.science/hal-01629220
}

Submitted on 6 Nov 2017

HAL is a multi-disciplinary open access archive for the deposit and dissemination of scientific research documents, whether they are published or not. The documents may come from teaching and research institutions in France or abroad, or from public or private research centers.
L'archive ouverte pluridisciplinaire HAL, est destinée au dépôt et à la diffusion de documents scientifiques de niveau recherche, publiés ou non, émanant des établissements d'enseignement et de recherche français ou étrangers, des laboratoires publics ou privés. 


\title{
Analysis of Charge Transport and Ionization Effect in Space-Used Polymers Under High-Energy Electron Irradiation
}

\author{
T. Paulmier, B. Dirassen, D. Payan, and M. Arnaout
}

\begin{abstract}
Polymer materials have been tested in the dedicated experimental facility SIRENE (ONERA, Toulouse, France) designed to reproduce the electron energy spectrum met in space in the $(0-400 \mathrm{keV})$ energy range and to perform electric analysis on the materials through dedicated protocols with potential, current, and contactless pulsed-electro acoustic (CNES patent) measurements. A Novel experimental approach shall as well be presented, which allowed bringing into evidence the complex response of polymers under irradiation: polarization and charge differential mobility as well as physical structural changes have been analyzed with these new techniques allowing a better understanding and prediction of charging behavior and radiation-induced conductivity evolution of these polymers under space conditions.
\end{abstract}

Index Terms-Charging, electric field, electron, ionization, model, radiation-induced conductivity (RIC), spacecraft, temperature.

\section{INTRODUCTION}

D IELECTRIC materials used on spacecraft are submitted to a large spectrum of electron radiation which induce the implantation of charged particles in their all thickness. This charge induce potential gradient which may trigger electrostatic discharges and/or secondary powered arc discharges on different parts of the satellite. Charging effect of space materials is, however, usually smoothed by ionization processes due to high-energy electron irradiation that enhances significantly the bulk electric conductivity in these materials: we speak about radiation-induced conductivity (RIC). It is of high importance to take into account this radiation effect for realistic predictions of spacecraft charging levels met in space environment. Other physical processes, such as polarization and interfacial effects, might play also a significant role on the charging behavior of space materials.

Polymers are widely used on spacecraft for thermal, optical, mechanical, or electrical application. These materials are very sensitive to radiation dose and their charging behavior

Manuscript received July 13, 2016; revised January 6, 2017; accepted February 7, 2017. This work was supported by CNES, F-31400 Toulouse, France, in the frame of successive R\&T projects.

T. Paulmier and B. Dirassen are with ONERA, The French Aerospace Laboratory, F-31055 Toulouse, France (e-mail: Thierry.Paulmier@onera.fr; Bernard.Dirassen@onera.fr).

D. Payan is with CNES, The French Space Agency, F-31400 Toulouse, France (e-mail: Denis.Payan@cnes.fr).

M. Arnaout is with Lebanese International University, Mazraa, Beirut 146404, Lebanon (e-mail: mohamad.arnaout@liu.edu.lb).

Digital Object Identifier 10.1109/TPS.2017.2681180 evolves quite swiftly with irradiation duration. A thorough analysis allowed demonstrating that RIC (instantaneous or delayed) depends not only on radiation dose, but also on total radiation dose, temperature, and electric field. The physical mechanisms steering these effects have been identified through a large experimental and parametric study. The attention has especially been focused on Kapton and fluorinated spaceused polymers. These mechanisms have been implemented in a physical model describing charge transport and ionization effect, based on solid-state physics. This paper presents both the experimental characterization results as well as numerical output that provided a support on the identification of these physical mechanisms.

\section{Experimental Setup and Protocol}

\section{A. SIRENE Irradiation Facility}

The experiments have been performed in the unique irradiation test facilities (SIRENE), funded by CNES and installed at ONERA (Toulouse, France) which allows charge characterization under geostationary (GEO)-like electron irradiation spectrum. Fig. 1 shows the electron beam spectral characteristics of the SIRENE facility with an energy spectrum $\left(K_{p}>5\right)$ approaching that of the GEO charging environment.

SIRENE electron spectrum $\left(\left[20 \mathrm{keV}, 250 \mathrm{pA} / \mathrm{cm}^{2}\right]+\right.$ [0-400 keV, $\left.50 \mathrm{pA} / \mathrm{cm}^{2}\right]$ ) experimental simulation is achieved with the use of two monoenergetic electron beams. In order to get a space like electron beam, the $400-\mathrm{keV}$ electron beam, passing through complex diffusion foils, is dispersed in energy from 0 to $400 \mathrm{keV}$. The nominal fluxes used for the $20-\mathrm{keV}$ monoenergetic beam and the distributed $400 \mathrm{keV}$ one are, respectively, equal to 250 and $50 \mathrm{pA} / \mathrm{cm}^{2}$ but can be raised, respectively, up to $1 \mathrm{nA} / \mathrm{cm}^{2}$ and $200 \mathrm{pA} / \mathrm{cm}^{2}$. A pumping system allows experiments at vacuum of around $10^{-6}$ mbar. The temperature of the sample holder can be controlled in the range of $-180{ }^{\circ} \mathrm{C}$ to $250{ }^{\circ} \mathrm{C}$ allowing to reproduce the temperature variation of materials on flight. The evolution of charging potential, during and after beam cutoff are monitored using a noncontact electrostatic probe (Kelvin probe TREK 3455ET) coupled with an electrostatic voltmeter (TREK 341B).

This irradiation facility allowed a thorough description of RIC dependence with applied parameters (radiation dose rate, total dose, temperature, and electric field). Recovery and aging processes have also been analyzed through dedicated 


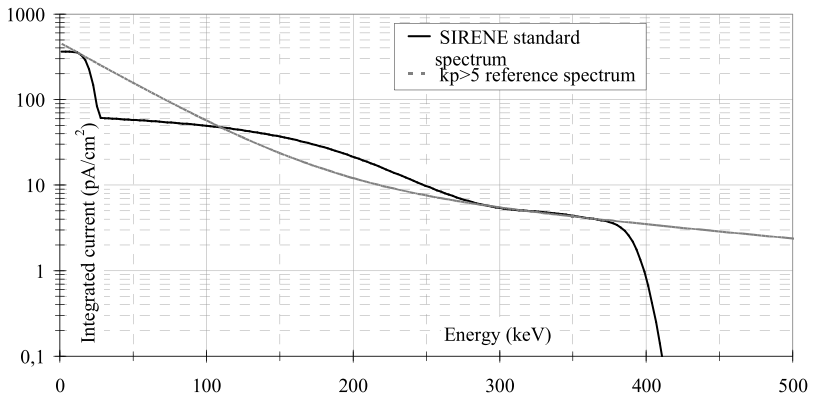

Fig. 1. SIRENE standard spectrum and reference $K_{P}>5$ spectrum.

experimental protocols allowing the optimization of physics model used for charging prediction in space environment.

\section{B. Experimental Setup and Protocol}

Studying the charging behavior of space polymer in space environment implies to perform thorough analysis on the ionization and charge transport processes in representative conditions. RIC depends on radiation dose, dose rate, temperature, and electric field [1]-[5]. Different experimental procedures have been devised at ONERA for the extraction of RIC and for studying the influence of the different above parameters. The first approach is the potential relation (PR) method. For this method, bulk induced conductivity is assessed through the analysis of the surface potential relaxation after charging the sample with low-energy electrons $(20 \mathrm{keV})$ up to a given surface potential [5]. During the electric potential relaxation, a 400-keV penetrating ionizing electron beam (which does not contribute to material charging) is used to stimulate the potential decay through the activation of RIC. To evaluate bulk conductivity of space materials, the material sample is modeled as a combination of a capacitance and resistance in parallel [6]. RIC can then be extracted from the following equation:

$$
\sigma_{\mathrm{RIC}}(t)=1 / V \cdot\left[(1-\psi-\beta) \cdot J_{0} \cdot L-\varepsilon \cdot d V / d t\right]
$$

for which $V$ is the absolute surface potential, $\psi$ is the total electron emission yield, $\beta$ is the leakage surface current coefficient, $L$ is the space charge distance to the ground, and $\varepsilon$ is the material permittivity.

Another conventional method is to measure the induced current at the rear face of an irradiated sample. This sample is composed of two electrodes on each side and a potential difference is applied between both electrodes. After annealing of the polarization current, a $400-\mathrm{keV}$ penetrating ionizing electron beam (which does not contribute to material charging) is used to stimulate the leakage current. From this current, we can extract the conductivity from the simple law

$$
\sigma=\frac{I}{V} \cdot \frac{L}{S}
$$

for which $I$ is the measured current, $V$ is the applied potential difference, $L$ is the thickness, and $S$ is the surface area of the sample.

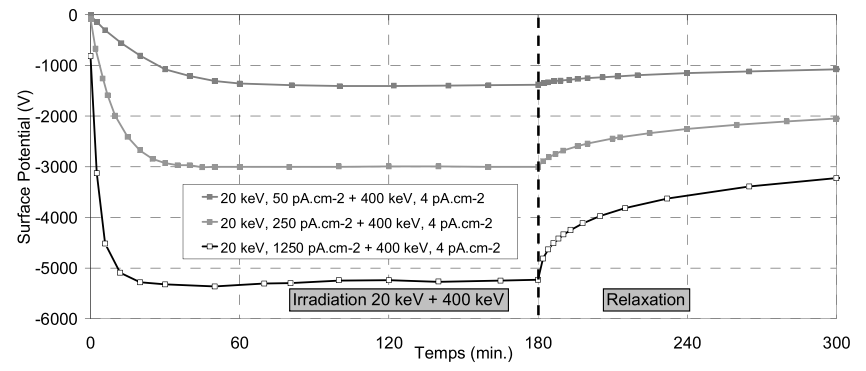

Fig. 2. Evolution of surface potential of Kapton HN $50 \mu \mathrm{m}$ irradiated with electrons at $400 \mathrm{keV}, 4 \mathrm{pA} \cdot \mathrm{cm}^{-2}$ and electrons at $20 \mathrm{keV}$ and different fluxes at (a) $50 \mathrm{pA} \cdot \mathrm{cm}^{-2}$, (b) $250 \mathrm{pA} \cdot \mathrm{cm}^{-2}$, and (c) $1250 \mathrm{pA} \cdot \mathrm{cm}^{-2}$.

This last method allows recording the conductivity at a constant potential and we are then able to discriminate between electric field effect and radiation dose effect.

\section{EXPERIMENTAL RESULTS}

\section{A. Effect of Electric Field on RIC}

The irradiation experiments allowed demonstrating that RIC of materials is strongly dependent on the applied electric field. The first experiments that brought into evidence this effect were performed on Kapton HN (thickness: $127 \mu \mathrm{m}$ ) irradiated with two electron beams at 20 and $400 \mathrm{keV}$ in energy: the first beam is used to charge the sample while the second one go through the sample, induces ionization and initiates RIC. Three different irradiation tests have been performed in which the $400-\mathrm{keV}$ beam flux was kept constant while we changed the 20-keV flux from one experiment to the other (Fig. 2) to adjust the electric field within the material. From Fig. 2, we can notice that increasing the $20-\mathrm{keV}$ charging flux by a factor of 5 or 25 does only yield an enhancement on the surface potential by a factor of 2 and 3.5. These experimental characteristics can be explained as follows: the electric field rise (due to the increase of charging flux) induces an increase of the RIC which counterbalances the charge implantation rise. In this first experiment, the induced electric field varies from $3 \times 10^{7}$ to $10^{8} \mathrm{~V} / \mathrm{m}$.

Dedicated tests on $25-\mu \mathrm{m}$ polyimide sample with the leakage current measurement method demonstrated as well that conductivity rises with the applied electric field when the electric field exceeds a threshold (Fig. 3). This method allows working at lower electric field that the previous potential measurement method. We can then observe saturation events on RIC that are difficult to observe with the PR method.

These results are in good agreement with the previous results coming out from the potential relaxation method [5], in regard of the increase of RIC with the electric field. The new result is the saturation of RIC at low electric field. These features have been ascribed to the effect of electric field on geminate recombination. For electric fields below a threshold, electric field is not sufficient to extract electronhole pairs and reduce recombination rate. Once the electric field exceeds this level, electron-hole pairs can be created and the ionization effective rate rises up. This behavior has already been identified by other researchers [7], [8]. From a 


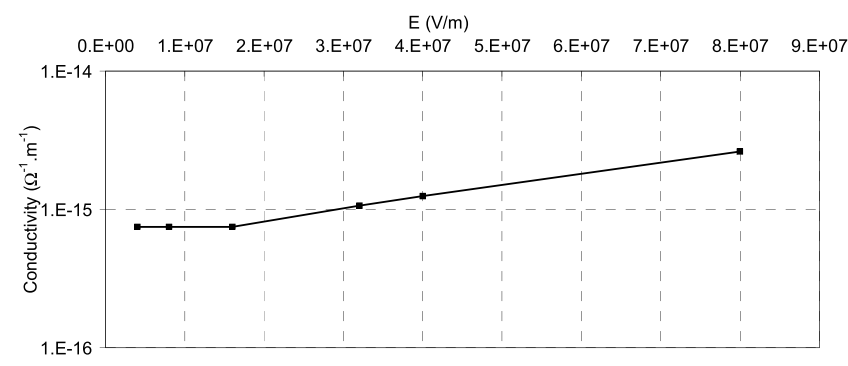

Fig. 3. Evolution of RIC measured by leakage current method on $25-\mu \mathrm{m}$ polyimide sample irradiated with $400 \mathrm{keV}, 4 \mathrm{pA} \cdot \mathrm{cm}^{-2}$ electron beam.

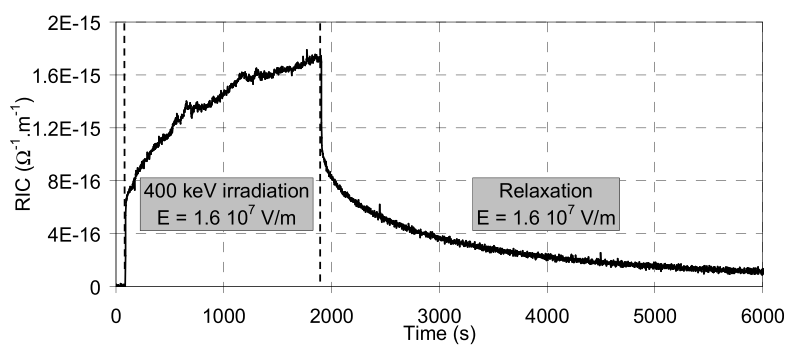

Fig. 4. Evolution of leakage current measured on 25- $\mu \mathrm{m}$ Kapton irradiated at $400 \mathrm{keV}, 4 \mathrm{pA} \cdot \mathrm{cm}^{-2}$ with an applied electric field of $1 \times 610^{7} \mathrm{~V} / \mathrm{m}$.

physical point of view, when the electric field or temperature are enhanced, the probability of electrons and holes getting separated increases (see (3), [9]), leading to a significant rise of the effective generation rate (4)

$$
P=\exp \left(-e^{2} / \varepsilon \cdot k \cdot T \cdot r_{0}\right) *\left(1+e^{3} \cdot E / 2 \varepsilon \cdot k^{2} \cdot T^{2}\right)
$$

$G(E, T)=g_{0} \cdot P(E, T)$.

\section{B. Effect of Radiation Dose on RIC and Analysis of Delayed RIC}

The leakage current measurement allowed demonstrating that RIC steadily rises with the increasing radiation dose (or irradiation duration), as can we see in Fig. 4. This observation is in good agreement with previous potential measurement of Kapton irradiated under representative electron spectrum (Fig. 5), for which we observed a continuous decline of the absolute surface potential that was already ascribed to RIC rise [5].

In Fig. 4, the 400-keV irradiation was switched off after a given duration $(1800 \mathrm{~s})$ while the electric field remained constant. This measurement first proves that the measured current is not due to polarization effect (since the measured current is mainly steered by irradiation). We can notice as well that RIC prevails for a long period of time after the irradiation was cutoff: this is due to delayed radiation induced conductivity (DRIC). Ionization effect keeps up for a long period of time due to electrons and holes detrapping. An other interesting and new feature observed with the leakage current method is the initial steep drop observed on DRIC after irradiation shut down followed by a slow decay. This characteristic has been modeled as well with the simple circuit model developed at ONERA [5] and is explained by a

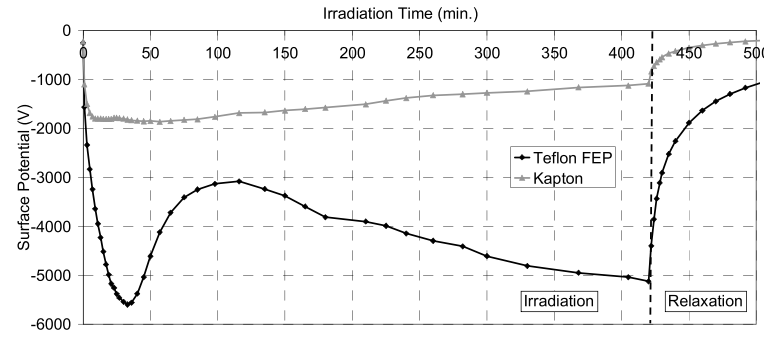

Fig. 5. Evolution of surface charging potential measured on $127-\mu$ m-thick Kapton and Teflon under GEO-like electron irradiation $\left(K_{p}>5\right)$-irradiation conditions: $\left(\left[20 \mathrm{keV}, 250 \mathrm{pA} \cdot \mathrm{cm}^{-2}\right]+\left[0-400 \mathrm{keV}, 50 \mathrm{pA} \cdot \mathrm{cm}^{-2}\right]\right)$.

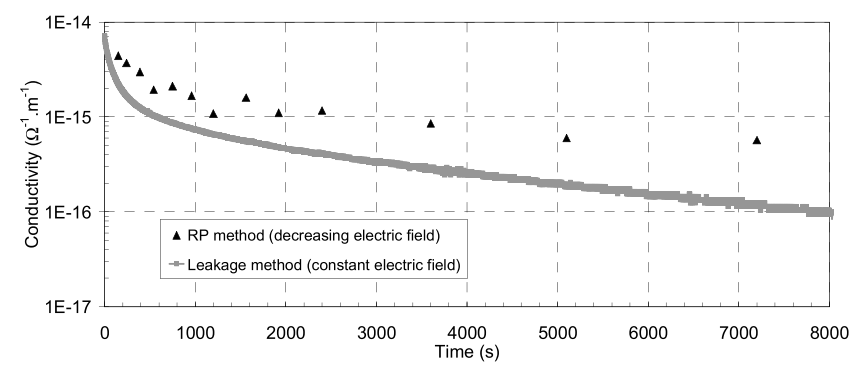

Fig. 6. Evolution of delayed RIC during relaxation on Kapton $\mathrm{HN}$ for an initial electric field (at the start of the relaxation) of $10^{7} \mathrm{~V} \cdot \mathrm{m}^{-1}$ assessed with two methods: the RP method for which electric field decreases along the relaxation process and the leakage current method for which electric field is constant along the process.

first rapid trapping of free charges followed by a continuous detrapping during relaxation.

Following the first initial drop, we have observed that the relaxation kinetics of DRIC measured with the leakage current method is higher than the one measured with the potential relaxation (PR) method (see Fig. 7). In the first method, the electric field is constant during the overall process, contrary to the PR method for which the electric field decreases along the relaxation process. This observation suggests that DRIC is electric field dependent, which could be ascribed to PooleFrenkel effect. For the leakage current method, the constant electric field allows a quicker release of trapped charges which are injected in the conduction band and then get recombined with trapped charges of opposite sign. The charge storage gets quickly lost contrary to the PR method for which the decreasing electric field allows a lower charge loss during relaxation and then a longer DRIC.

RIC has been characterized as well on fluorinated ethylene propylene (FEP) sample with the leakage current method at constant electric field under successive irradiation. We can notice a nonmonotonic evolution of RIC along irradiation that was already identified to account for the potential evolution observed under representative electron spectrum (Fig. 5) [6]. This material is submitted first to an increase of its RIC followed by a continuous decline of this parameter along the irradiation. The second irradiation presents a lower RIC and we have already demonstrated that this behavior is due to the effect of charges trapped during the first irradiation which act as recombination centers during the second irradiation process. Finally, we can also notice a delayed RIC that steeply drops down during relaxation. 


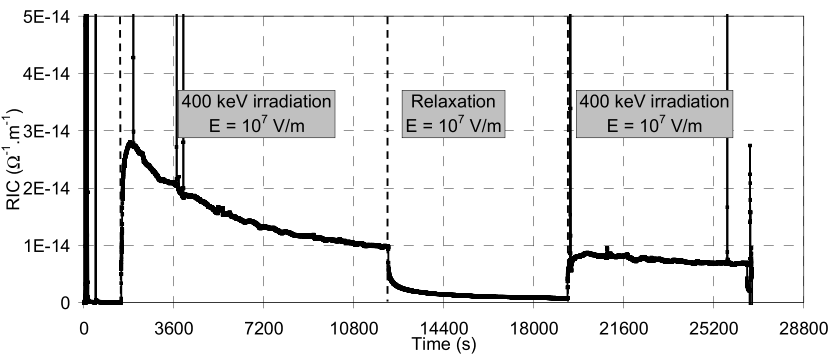

Fig. 7. Evolution of leakage current measured on $100-\mu \mathrm{m}$ FEP sample irradiated at $400 \mathrm{keV}, 10 \mathrm{pA} \cdot \mathrm{cm}^{-2}$ with an applied electric field of $10^{7} \mathrm{~V} / \mathrm{m}$.

\section{NumericAl ANALYSis}

A dedicated physical and numerical model, called circuit model, has been developed at ONERA to describe the evolution of surface potential and RIC of space-used materials irradiated with high-energy electrons and representative charging conditions. This model is based on former studies and models devised by Rose [2] and Fowler [1].

Two levels of localized traps have been implemented in this model: one deep trap for which detrapping should be very low but should act as recombination centers if filled with electrons; a second trap corresponding to middle energy depth and for which detrapping is easier. Shallower traps are supposed to get in thermal equilibrium with the extended state. Mobility for free charges is therefore an average mobility taking into account this thermal equilibrium process. We assume as well that a free charge recombines with a localized charge with opposite sign. Based on the above assumptions and introducing charge detrapping process in the model, the equations describing the circuit RIC model are

$$
\begin{aligned}
\frac{d n}{d t}= & g(E, T)-\alpha_{1} n\left(p_{t 1}+p_{t 2}\right) \\
& -\frac{n}{\tau_{n 1}}-\frac{n}{\tau_{n 2}}+\frac{n_{t}}{\tau_{n t 1}}+\frac{n_{t 2}}{\tau_{n t 2}} \\
\frac{d p}{d t}= & g(E, T)-\alpha_{2}\left(n_{t 1}+n_{t 2}\right) p \\
& -\frac{p}{\tau_{p 1}}-\frac{p}{\tau_{p 2}}+\frac{p_{t}}{\tau_{p t 1}}+\frac{p_{t 2}}{\tau_{p t 2}} \\
\frac{d n_{t 1}}{d t}= & \frac{n}{\tau_{n 1}}-\frac{n_{t 1}}{\tau_{n t 1}}-\alpha_{2} p n_{t 1} \\
\frac{d n_{t 2}}{d t}= & \frac{n}{\tau_{n 2}}-\frac{n_{t 2}}{\tau_{n t 2}}-\alpha_{2} p n_{t 2} \\
\frac{d p_{t 1}}{d t}= & \frac{p}{\tau_{p 1}}-\frac{p_{t 1}}{\tau_{p t}}-\alpha_{1} n p_{t 1} \\
\frac{d p_{t 2}}{d t}= & \frac{p}{\tau_{p 2}}-\frac{p_{t 2}}{\tau_{p t 2}}-\alpha_{1} n p_{t 2}
\end{aligned}
$$

for which $g(E, T)$ is the electron /hole pairs generation rate which varies with the applied electric field and temperature [according to the Onsager theory (geminate recombination)], $\alpha_{1}$ and $\alpha_{2}$ are the recombination rates, $n, n_{t 1}$, and $n_{t 2}$ are densities of free and trapped electrons, $p, p_{t 1}$, and $p_{t 2}$ are densities of free and trapped holes, $\tau_{n 1}, \tau_{n 2}, \tau_{p 1}$, and $\tau_{p 2}$ are trapping time for free electrons and holes, $\tau n_{t 1}, \tau n_{t 2}, \tau p_{t 1}$, and $\tau p_{t 2}$ are detrapping time for trapped electrons and holes. In this model, the effect of temperature on detrapping time

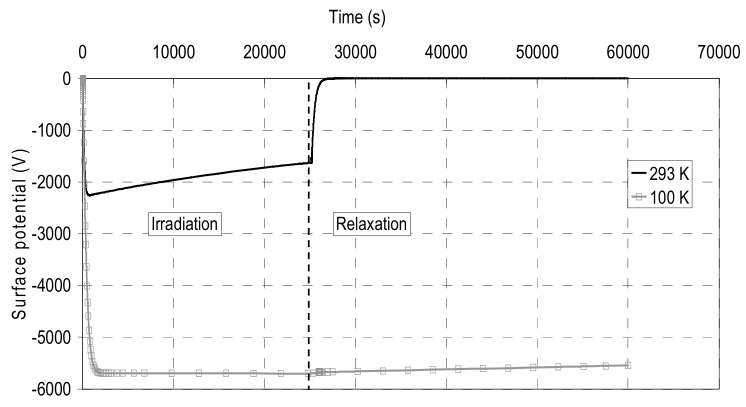

Fig. 8. Evolution of surface potential, assessed numerically, on $127-\mu \mathrm{m}$ thick Kapton irradiated under GEO-like electron irradiation at two different temperature levels (100 and $293 \mathrm{~K}$ ).

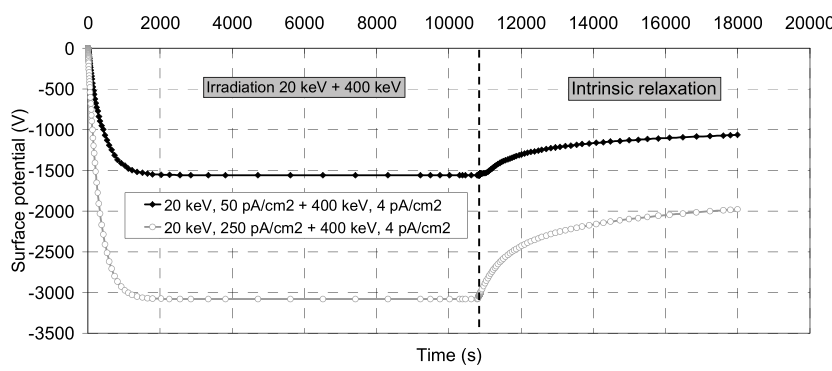

Fig. 9. Evolution of surface potential, assessed numerically, on 50- $\mu \mathrm{m}$-thick Kapton irradiated with electrons at $400 \mathrm{keV}, 4 \mathrm{pA} \cdot \mathrm{cm}^{-2}$ and electrons at $20 \mathrm{keV}$ and different fluxes at (a) $50 \mathrm{pA} \cdot \mathrm{cm}^{-2}$ and (b) $250 \mathrm{pA} \cdot \mathrm{cm}^{-2}$.

has been taken into account through a conventional Arrhenius law that reflects the fact that detrapping kinetics is enhanced with the increasing temperature

$$
\tau_{t}=\tau_{t 0} \cdot \exp \left(-\frac{E_{a}}{k \cdot T}\right) .
$$

We can then assess densities of free electrons and holes that are used in (10) for surface potential assessment

$$
\frac{d V}{d t}=\frac{L j(1-\eta-\beta)-e\left(\mu_{n} n+\mu_{p} p\right) V}{\varepsilon}
$$

in which $L$ is the sample thickness, $J$ is the incident flux of low-energy implanted electrons $(20 \mathrm{keV}), \eta$ is the secondary electron emission yield, $\beta$ is the surface leakage parameter, $V$ is the surface potential, $e$ is the elementary charge, and $\varepsilon$ is the material permittivity.

This circuit model has been validated on $127-\mu$ m-thick Kapton irradiated under GEO-like electron irradiation at different temperature levels. Fig. 8 presents the evolution of surface potential on this material at 100 and $293 \mathrm{~K}$. These results are in good agreement with the experimental results with the following trends:

1) a shift of the surface potential to lower values with the decreasing temperature;

2) a decline of RIC increase during irradiation when temperature drops down;

3) a decline of potential relaxation kinetics with temperature.

Fig. 9 also presents as well the evolution of surface potential assessed numerically when the material is irradiated by two electron beams at 20 and $400 \mathrm{keV}$. The $20-\mathrm{keV}$ charging flux as been changed from one run to the other while the 
400- keV flux remained constant (the same situation as in Fig. 2). We are able to reproduce with this circuit model the same trends as observed experimentally with a surface potential that do not evolves linearly with the increasing charging flux. Relaxation kinetics is not so satisfactory and the reason is that we need to implement the Poole-Frenkel effect in the model to be more realistic.

\section{CONCLUSION}

This paper demonstrates the specific behavior of space polymers and their conduction processes under high-energy electron irradiation. RIC is indeed steered by several physical processes in competition and is therefore dependent upon radiation dose rate, total received radiation dose, electric field, and temperature. These different effects have been brought into evidence at ONERA through dedicated experimental procedures. They have been implemented as well in a physical and numerical model that allows understanding and predicting the charging behavior of space dielectrics in representative space radiation conditions. This model has been validated with the large experimental database available at ONERA.

\section{ACKNOWLEDGMENT}

The authors would like to thank CNES, the French Space Agency, for technical and scientific support over the successive R\&T studies.

\section{REFERENCES}

[1] J. F. Fowler, "X-ray induced conductivity in insulating materials" Proc. R. Soc. Lond. A, Math. Phys. Sci., vol. 236, no. 1207, pp. 464-480, 1956.

[2] A. Rose, "Recombination processes in insulators and semiconductors," Phys. Rev., vol. 97, pp. 322-333, Jan. 1955.

[3] B. Gross, R. M. Faria, and G. F. Ferreira, "Radiation induced conductivity in Teflon irradiated by X-rays," J. Appl. Phys., vol. 52, no. 2, pp. 571-578, 1981.

[4] A. P. Tyutnev, V. S. Saenko, I. A. Smirnov, and E. D. Pozhidaev, "Radiation-induced conductivity in polymers during long-term irradiation," High. Energy Chem., vol. 40, no. 5, pp. 319-330, 2006.

[5] T. Paulmier, B. Dirassen, M. Arnaout, D. Payan, and N. Balcon, "Radiation-induced conductivity of space used polymers under high energy electron irradiation," IEEE Trans. Plasma Sci., vol. 43, no. 9, pp. 2907-2914, Sep. 2015.

[6] R. Hanna et al., "Radiation induced conductivity in Teflon FEP irradiated with multienergetic electron beam," IEEE Trans. Plasma Sci., vol. 41, no. 12 , pp. $3520-3525$, Dec. 2013.

[7] R. G. Filho, B. Gross, and R. M. Faria, "Induced conductivity of mylar and Kapton irradiated by X-rays," IEEE Trans. Electr. Insul., vol. 21 , no. 3, pp. 431-436, Jun. 1986.

[8] R. S. Ikhsanov, A. P. Tyutnev, V. S. Saenko, and E. D. Pozhidaev, "A theoretical description of geminal electrical conductivity in the approximation of isolated ion pairs on the basis of a solution to the Smoluchowski equation," Russ. J. Phys. Chem. B, vol. 2, no. 2, pp. 309-314, 2008.

[9] R. H. Barlett, G. A. Fulk, R. S. Lee, and R. C. Weingart, "Temperature dependence of X-ray-induced photoconductivity in Kapton and Teflon," IEEE Trans. Nucl. Sci., vol. 22, no. 6, pp. 2273-2276, Dec. 1975. 